\title{
Effectiveness of Eccentric Exercise for the Management of Rotator Cuff Tendinopathy: A Critical Review
}

\author{
Zhiwei Yang* and Sheng Wang \\ Suzhou Science \& Technology Town Hospital of Nanjing Medical University, Suzhou, China
}

*Corresponding author: Zhiwei Yang, The affiliated Suzhou Science \& Technology Town Hospital of Nanjing Medical University, Suzhou, China

\section{Introduction}

The rotator cuff (RC) mainly contains four muscles and their tendons: infraspinatus, supraspinatus, teres minor and the subscapularis [1]. These four muscles and their tendons surround the shoulder joint [2]. It is a group of muscles start from the scapular and attach around the humeral head, forming a cuff at the anatomical neck of the humeral head. The main function of the RC is to stabilize and activate the glenohumeral joint (ibid). The RC primarily performs three types of movement: abduction, external rotation and internal rotation. Previous studies report that there is no strong evidence that the RC contributes to glenohumeral movement (ibid). However, there are a large number of published studies that describe the link between RC and glenohumeral joint stability [3]. Hence, RC injury can result in loss of stabilization of the glenohumeral joint. RC tendinopathy (RCT) has been regarded as a kind of RC injury. RCT is a term used to describe a combination of pain and weakness in the RC tendon [4]. Also, it can be loosely described as excessive load resulting in impaired performance of the RC tendon when experienced with particular movements of the shoulder [5]. The prevalence of RCT is $14 \%$ in the general labor population [6]. In addition, a number of authors have considered the prevalence of RCT. It has been reported that prevalence is 19\% among all ages (ibid). Therefore, the high prevalence of RCT cannot be underestimated. The diagnosis of RCT can be measured by ultrasound and magnetic resonance imaging (MRI). Its diagnosis also can be considered in association with pain symptoms [7-9].

\section{Pathophysiology}

There are many risk factors that can cause RC injuries. These include glenohumeral joint dynamic instability, joint abnormality and abnormal shoulder structure [10]. After a RC injury, the damaged tendon gradually develops microtears, calcification and fiber vascular proliferation [11]. The pathophysiology of RC injury can be attributed to the result of series of inflammatory reactions after soft tissue injury. However, RCT is characterized by different types of RC tendon injury. Tendinopathy is a common term used to describe pathological changes in tendons. Previous research has established that the pathology of RCT primarily contains intrinsic mechanisms, extrinsic mechanisms or combined mechanisms [2-7]. Intrinsic mechanisms can be defined as pathological changes directly affecting the tendon itself [12]. Excessive load is an important contributor to RC intrinsic tendinopathy. Healthy adult's RC tendons consist of water, collagen, proteoglycan and glycosaminoglycan [13]. The largest proportion of RC tendon is type I collagen [14]. The collagen fibers of the tendons are confluent and form a cuff on the humeral head. It also forms a five-layer complex near the greater tuberosity. The first to fifth layers are superficial coracohumeral ligament, the main portion of tendon complex, dense fascicles, loose connective tissue and fibrocartilage, respectively. This composite structure seems to have excellent stability and elasticity. However, according to the intrinsic theory, the transformation of collagen bundles density and orientation could result in sheer forces between layers (ibid). Excessive load can cause changes and result in a strong force in the tendon. This increases with age and the impact of the disease can cause degenerative changes which can reduce adaptability in the tendon. There is an unambiguous relationship between degenerative changes and increasing age [15]. Excessive load plus degenerative changes lead to variation in biology and morphology of the RC tendon (ibid). There is a relatively small body of literature that is concerned with degenerative changes in RC tendons which contained calcification, fatty infiltration, tendon thinning and vascular proliferation [16]. Moreover, in an investigation into degenerative changes, [17] found that type III collagen has been increased that could decrease the elasticity of tendon during RC injury. Extrinsic mechanisms can broadly be defined as RC tendon injury that is potentially attributed to the friction on impact with external structures. These structures contain the humeral head and coracoacromial arch. Numerous studies have attempted to explain that $95 \%$ of RC injury was caused by impingement between the coracoacromial arch and humeral head [18]. Even in normal shoulder anatomy, a series of shoulder functions such as elevation 
and abduction can damage the $\mathrm{RC}$ tendon. There are a large number of published studies that describe the link between external impingement and RCT $[19,20]$. It has been thought that extrinsic impingement primarily results in pathological changes near the glenohumeral joint side of the RC tendon. The causes of intrinsic and extrinsic mechanisms are different, but both of them lead to an inflammatory response. After RCT, tendon healing is commonly divided into three parts [21, 22].

Firstly, the inflammatory phase lasts for one week. During this phase, vascular permeability increases, lymph and inflammatory cells flood into the lesion site. Secondly, in the proliferative phase, the influx of inflammatory cells produces some growth factors and cytokines which result in recruitment and proliferation of fibroblasts. Finally, in the remodeling phases, fibroblasts proliferate and begin to generate, orient, deposit and interconnect than become collagen [23] has shown that intrinsic factors or extrinsic factors alone do not increase the prevalence of RCT. In total 108 mature rats were equally divided into three groups: extrinsic compression group, overuse group and a combination of overuse and extrinsic compression group. Results from the studies indicated that no significant differences were found between separate overuse or extrinsic compression and RCT. Also, a positive correlation was found between a combination of overuse and extrinsic compression and RCT. This study suggested that a combination of intrinsic factors (overuse) and extrinsic factors could increase the possibility of RCT. However, these results came from study on animals and are difficult to directly utilize in clinical conditions. This is because human beings and animals have different anatomical and biological characteristics.

\section{The Physiological Effects of Eccentric Exercise}

Eccentric exercise is a type of muscle action characterized by contraction of a muscle during its lengthening due to an external load. To determine the effect of eccentric training, [24]. carried out an experiment. This study recruited 12 young male soccer players (mean $=26$ years) and implemented 3 sets of heels raising eccentric training twice a day for 12 weeks. Six participants have Achilles tendinosis and others were healthy one. To evaluate collagen synthesis and degradation, blood samples were taken 1 week before and after 12-week eccentric training programme through micro dialysis technique. Pain visual analogue scale (P-VAS) also was used to assess the level of pain before and after 12-week training. There are a large number of publish studies that describe the link between carboxyterminal propertied of type I collagen (PICP) increasing in blood and type I collagen synthesis. Also, data from several sources have identified the increasing carboxyterminal telopeptide region of type I collagen (ICTP) associated with the degeneration of type I collagen $[25,26]$. The findings of this study showed that PICP increased significantly in the blood after 12 weeks of training in injured tendon, but not changed in healthy tendon. ICTP have no significant difference before and after study in both health and injured. Many recent studies have shown the same findings as [23] experiment $[27,28]$. To eliminate bias, this study carried out the same starting time (9am) and controlled the room temperature $\left(25{ }^{\circ} \mathrm{C}\right)$. In injured group, the level of pain also reduced after 12 weeks training. Combining the outcomes from blood samples and P-VAS, this study could provide strong evidence to support the effectiveness of eccentric exercise. However, although the study examined plasma concentrations of PICP and ICTP, the changes of collagen type I formation could not directly be observed. Previous studies suggested that MRI or ultrasound can observe the changes in collagen type I formation [29,30]. More medical examinations should be included in further studies. Also, the population of this study is professional athletes, instead of ordinary people. It may reduce the generalizability of this study. Further studies should focus on the ordinary population and carry out more clinical examinations.

One of the most influential accounts of eccentric training came from [31]. The findings of this study demonstrated significant neovascularization after eccentric training. 22 males and 8 females with Achilles tendinopathy participated in this investigation. Ultrasonography and colored Doppler were used to examine the neovascularization in the tendon before and after intervention. Participants were asked to complete 12 weeks of knee eccentric exercise twice per day, seven days per week. The result of this study indicated that eccentric training is likely to accelerate the neovascularization. Although this study focused on patients with Achilles tendinopathy, the benefit of eccentric exercise is likely to generalize to other tendinopathies, due to the similar anatomical, physiological and pathological feature of tendinopathies [32]. However, different gender ratios (22 males and 8 females) may result in bias of experimental outcomes. Compared to women, men are more likely to regenerate new blood vessels in the tendon area after eccentric training [33]. This is due to the difference in the physiological characteristics of men and women. In addition, there is no control group in this study. Although setting a control group is not ethically permissible, a control group could reduce cointervention bias and make the experimental results more reliable and valid. A systematic review that is often cited in research on the effect of eccentric exercise is that of [34] who found that eccentric training may promote increases in tendon stiffness. This study also reported that low intensity eccentric exercise is more effective than concentric exercise for increasing a tendon's mechanical tension. The keywords "eccentric", "tendon" and "performance" were used in its search strategy. 40 studies were included and total of 1150 participants (406 women and 744 men) were recruited in this study. Results of this study found that, compared to concentric exercise, muscle cross-sectional area (CSA) increases significant difference after eccentric exercise. Eccentric exercise can promote more CSA synthesis than concentric exercise [35]. also identified that increasing number of CSA were beneficial for the stiffness of tendons. In addition, this study highlighted low intensity eccentric exercise was beneficial for the maximal tendon force and stress, instead of high intensity eccentric exercise. The whole design of this study is rigorous and appropriate, given proper keywords and suitable search strategy. However, this study carried out narrative analysis which cannot provide strong evidence for the findings. In addition, the study recruits healthy participants which may not demonstrate the effect of eccentric training in clinical condition. Therefore, these findings should be translated with caution into 
clinical scenarios. Overall, these studies provided relevant evidence that eccentric exercise promote collagen synthesis, formation of new blood vessels and increases tendon stiffness. However, further study should focus on clinical condition and more rigorous experimental design.

\section{The Effectiveness of Eccentric Exercise}

Reported [36] positive correlation between tendon repairing and supraspinatus and infraspinatus eccentric exercise. Ten middleaged (mean age $=54$ ) patients with diagnosed RCT were recruited from two health care clinics. All the patients were provided with shoulder abduction and external rotation eccentric training which involved 90 repetitions per day. Shoulder pain was assessed by visual analogue scale (VAS) after the training programme, which was believed to be valid and reliable for evaluating level of pain. According to the result of this study, VAS significantly reduced from 57 to 29. Authors attributed this improvement to healing of tendon. This hypothesis seems to be consistent with the study of [23]. which found significantly improved collagen synthesis after eccentric training. In Susanne's study, ten middle-aged participants indicated small sample size and low generalizability. However, compared to middle aged people, the synthesis of young people's collagen is significantly faster [37-39]. found that insulin-like growth factors that promote collagen synthesis increased significantly among young people (mean age $=25$ ) compared with elderly people (mean age 70 years). The design of this study is a single-subject research design which is appropriate for the study condition. Therefore, the outcomes produced by middle-aged people as research participants can be accepted. [40] investigated the effectiveness of eccentric training comparing with conventional exercise in clinical RCT. 36 patients diagnosed with RCT, were recruited and randomly divided into eccentric exercise group (20) and conventional exercise group (16).

The eccentric exercise group (mean age $=50.2$ ) performed external rotator and abduction exercises for the shoulder. The conventional exercise group (mean age $=48.9$ ) contained shoulder shrugs and stretching exercise for the pectoralis. Both groups completed 12 weeks of daily exercise at home. 14 weeks after the training programme $\left(26^{\text {th }}\right.$ week), the Constant Murley Score (CMS) and Pain VAS were applied for measuring shoulder function and pain. Results of this study indicated that both eccentric and conventional exercise for the $\mathrm{RC}$ area improve significantly in shoulder function and pain after 26 weeks. The findings from this study also shown that there is no significant difference between eccentric training and conventional training for managing shoulder pain and function after 26 weeks. This study provided 12 weeks training and follow-up after 26 weeks, giving plenty time to examine the results. However, single-blinded instead of doubleblinded controlled trial measures were implemented in this study. This may lead to experimenter bias. In addition, although different language versions of the CMS showed good reliability and validity $[41,42]$, this instrument needs to have established standards [43]. Therefore, this study showed that in the long term, both eccentric and conventional exercise is beneficial for middle-aged people with RCT.
There is a published study describing the role of eccentric exercise for managing RCT [44]. A total of 11 patients with diagnosed RCT and preparing for arthroscopic subacromial decompression surgery were recruited in this study. All patients were randomly divided into 3 groups: control group, concentric $\mathrm{RC}$ exercise group and eccentric RC exercise group. The concentric group was asked to perform shoulder abduction exercises, 3 sets of 15 repetitions twice a day from $0^{\circ}$ to $90^{\circ}$. The eccentric group was asked to perform shoulder adduction exercises, 3 sets of 15 repetitions twice a day from $90^{\circ}$ to $0^{\circ}$. The control group were asked to carry out standard practice without normal treatment pathway. The Oxford shoulder Score and pain Visual Analogue Scale were used to measure shoulder function and pain at the beginning, after 4 weeks and after 8 weeks. Interestingly, results from this study did not indicate any statistically significant differences between all three groups. In addition, two individuals in the eccentric group determined to cancel the surgery and continue exercise at the end of study. Compared to [37]. study, a total of 8 weeks length of this study is relatively short. It may demonstrate that longer study could result in statistically significant differences [45-47] also found that a longer follow-up period of eccentric exercise can avoid shoulder surgery. To compare with eccentric exercise and concentric exercise, further study design should extend the length of the experiment or implement a follow-up period. However, 11 patients is a small sample size which cannot provide strong evidence to support the outcomes of the study. In addition, although individual cases show significant improvement and avoid surgery after this study, this result may not be generalizable. Overall, data from this study gave no significant differences between concentric exercise and eccentric exercise on managing RCT. Moreover, short-term eccentric exercise may not have obvious therapeutic effect. Further study should collect more samples and extend experimental time.

\section{Conclusion}

Overall, the present study was designed to determine the effect of eccentric exercise. The research findings clearly indicated that eccentric exercise can promote the synthesis of type I collagen, accelerate the neovascularization and increase the integrity of a tendons. The second aim of this study was to investigate the effectiveness of eccentric exercise on RCT. Generally, the investigations have shown that, in the long term, eccentric, concentric and conventional exercise are beneficial for patients with RCT. Compared to concentric and conventional exercise, although eccentric exercise may not have obvious therapeutic effect, evidence indicated that, in the long term, eccentric exercise should be more effective in managing RCT than other treatments. However, the findings in this assignment are subject to at least three limitations. First, different gender ratio and age stage could lead to bias. Secondly, small sample size could decrease the generalizability. Finally, single examinations method and lack of randomization show that results should be interpreted with caution. Nonetheless, therapists may still select eccentric exercise or combine them with other treatment to manage RCT in clinical condition. Further investigation should increase sample size, balance gender ratio and use diverse examination methods. Also, it is necessary to determine 
which level of intensity of eccentric exercise is more effective in treating RCT. In addition, accurate eccentric exercise should be investigated as the RC contains four muscles.

\section{References}

1. Matthew DM (2014) Rotator Cuff Repair: Published Evidence on Factors Associated with Repair Integrity and Clinical Outcome. The American Journal of Sports Medicine 43(2): 491-500.

2. Lewis JS (2009a) Rotator cuff tendinopathy. British Journal of Sports Medicine 43(4): 236.

3. Lewis JS (2009b) Rotator cuff tendinopathy/subacromial impingement syndrome: is it time for a new method of assessment? British Journal of Sports Medicine 43(4): 259-264

4. Gomberawalla MM, Sekiya JK (2014) Rotator Cuff Tear and Glenohumeral Instability: A Systematic Review. Clinical Orthopaedics and Related Research 472(8): 2448-2456.

5. Lorenz D (2011) Shoulder tendinopathy. Physical Therapy Reviews 16(5): 365-373.

6. Toliopoulos P (2014) Efficacy of surgery for rotator cuff tendinopathy: a systematic review. Clinical Rheumatology 33(10): 1373-1383.

7. Littlewood C (2013) Development of a self-managed loaded exercise programme for rotator cuff tendinopathy. Physiotherapy 99(4): 358 362.

8. Lee SU (2016) Real-time sonoelastography in the diagnosis of rotator cuff tendinopathy. Journal of Shoulder and Elbow Surgery 25(5): 723 729.

9. Seitz AL (2011) Mechanisms of rotator cuff tendinopathy: Intrinsic, extrinsic, or both? Clinical Biomechanics 26(1): 1-12.

10. Su X (2018) Effects of high- and low-energy radial shock waves therapy combined with physiotherapy in the treatment of rotator cuff tendinopathy: a retrospective study. Disability and Rehabilitation 40(21): 2488-2494.

11. Omoumi P (2010) Glenohumeral joint instability. Journal of Magnetic Resonance Imaging 33(1): 2-16.

12. Rowshan K (2010) Development of Fatty Atrophy After Neurologic and Rotator Cuff Injuries in an Animal Model of Rotator Cuff Pathology. J Bone Joint Surg Am 92(13): 2270-2278.

13. Neviaser A (2012) Basic mechanisms of tendon fatigue damage. Journal of Shoulder and Elbow Surgery 21(2): 158-163.

14. Koca TT(2017) Clinical features of patients diagnosed with degenerative rotator cuff tendon disease: a 6-month prospective-definitive clinical study from turkey. Journal of Physical Therapy Science 29(8): 1433-1437.

15. Malcarney HL, Murrell GAC (2003) The Rotator Cuff. Sports Medicine 33(13): 993-1002.

16. Hashimoto T (2003) Pathologic Evidence of Degeneration as a Primary Cause of Rotator Cuff Tear. (415):111-120.

17. Riley GP (1994) Tendon degeneration and chronic shoulder pain: changes in the collagen composition of the human rotator cuff tendons in rotator cuff tendinitis. Annals of the Rheumatic Diseases 53(6): 359366.

18. Vincent K (2017) Are degenerative rotator cuff disorders a cause of shoulder pain? Comparison of prevalence of degenerative rotator cuff disease to prevalence of nontraumatic shoulder pain through three systematic and critical reviews. Journal of Shoulder and Elbow Surgery 26(5): 766-773.

19.Riand N (1998) Results of derotational humeral osteotomy in posterosuperior glenoid impingement. The American journal of sports medicine $26(3): 453-459$.
20. Jobe CM (1997) Superior Glenoid Impingement. Orthopedic Clinics 28(2): 137-143.

21. Zhaozhu L (2004) Inflammatory Response of Human Tendon Fibroblasts to Cyclic Mechanical Stretching. The American Journal of Sports Medicine 32(2): 435-440

22. Jason LD (2012) Comparison of the Acute Inflammatory Response of Two Commercial Platelet- Rich Plasma Systems in Healthy Rabbit Tendons. The American Journal of Sports Medicine 40(6): 1274-1281.

23. Manning Cionne N (2014) The early inflammatory response after flexor tendon healing: A gene expression and histological analysis. Journal of Orthopaedic Research 32(5): 645-652.

24. Soslowsky LJ (2002) Rotator Cuff Tendinosis in an Animal Model: Role of Extrinsic and Overuse Factors. Annals of Biomedical Engineering 30(8): 1057-1063.

25. Langberg H (2007) Eccentric rehabilitation exercise increases peritendinous type I collagen synthesis in humans with Achilles tendinosis. Scandinavian Journal of Medicine \& Science in Sports 17(1): 61-66.

26. Melkko J (1990) Radioimmunoassay of the carboxyterminal propeptide of human type I procollagen. Clinical Chemistry 36(7): 1328-1332.

27. Gurmarnik S (1996) A combination of low dose spinal and general anaesthesia for laparoscopic cholecystectomy. Canadian Journal of Anaesthesia 43(1): 95-95

28. Koskinen SO (2002) Short-term effects of forced eccentric contractions on collagen synthesis and degradation in rat skeletal muscle. Pflügers Archiv 444(1): 59-72.

29. Burd NA (2010) Low-Load High Volume Resistance Exercise Stimulates Muscle Protein Synthesis More Than High-Load Low Volume Resistance Exercise in Young Men. PLoS ONE 5(8): e12033.

30. Sun Y (2014) In Vivo Magnetic Resonance Imaging of Type I Collagen Scaffold in Rat: Improving Visualization of Bladder and Subcutaneous Implants. Tissue Engineering Part C: Methods 20(12): 964-971.

31. Yrineo AA (2017) Murine ultrasound-guided transabdominal paraaortic injections of self- assembling type I collagen oligomers. Journal of Controlled Release 249: 53-62.

32. van den Oord SCH (2014) Impact of gender on the density of intraplaque neovascularization: A quantitative contrast-enhanced ultrasound study. Atherosclerosis 233(2): 461-466.

33. Douglas J (2017) Chronic Adaptations to Eccentric Training: A Systematic Review. Sports Medicine 47(5): 917941.

34. Jelinsky SA (2011) Regulation of gene expression in human tendinopathy. BMC Musculoskeletal Disorders 12: 86-86.

35. Öhberg L, Alfredson H (2004) Effects on neovascularisation behind the good results with eccentric training in chronic mid-portion Achilles tendinosis? Knee Surgery Sports Traumatology Arthroscopy 12(5): 465470.

36. Ryan Eric D (2009) Passive properties of the muscle-tendon unit: The influence of muscle cross- sectional area. Muscle \& Nerve 39(2): 227229.

37. Susanne B (2011) Evaluation of an exercise concept focusing on eccentric strength training of the rotator cuff for patients with subacromial impingement syndrome. Clinical Rehabilitation 25(1): 69- 78.

38. Carroll CC (2008) Influence of aging on the in vivo properties of human patellar tendon. Journal of Applied Physiology 105(6): 1907-1915.

39. Kostrominova TY, Brooks SV (2013) Age-related changes in structure and extracellular matrix protein expression levels in rat tendons. Age 35(6): 2203-2214. 
40. Nielsen RH (2013) Increase in tendon protein synthesis in response to insulin-like growth factor- I is preserved in elderly men. Journal of Applied Physiology 116(1): 42-46.

41. Dejaco B (2017) Eccentric versus conventional exercise therapy in patients with rotator cuff tendinopathy: a randomized, single blinded, clinical trial. Knee Surgery Sports Traumatology Arthroscopy 25(7): 2051-2059

42. Çelik D (2016) Turkish version of the modified Constant-Murley score and standardized test protocol: reliability and validity. Acta Orthop Traumatol Turc 50(1): 69-75.

43. Moeller AD (2014) The Danish version of the modified Constant-Murley shoulder score: reliability, agreement, and construct validity. Journal of Orthopaedic \& Sports Physical Therapy 44(5): 336-335.
44. Roy JS (2010) A systematic review of the psychometric properties of the Constant-Murley score. Journal of Shoulder and Elbow Surgery 19(1): 157-164.

45. Marcus B, Nicola A (2014) A randomised controlled feasibility study investigating the use of eccentric and concentric strengthening exercises in the treatment of rotator cuff tendinopathy. SAGE Open Medicine 2: 2050312113520151.

46. Jonsson P (2006) Eccentric training in chronic painful impingement syndrome of the shoulder: results of a pilot study. Knee Surgery Sports Traumatology Arthroscopy 14(1): 76-81.

47. Walch G (1992) Impingement of the deep surface of the supraspinatus tendon on the posterosuperior glenoid rim: an arthroscopic study. Journal of shoulder and elbow surgery 1(5): 238- 245.

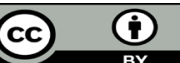

This work is licensed under Creative Commons Attribution 4.0 License

To Submit Your Article Click Here:

Submit Article

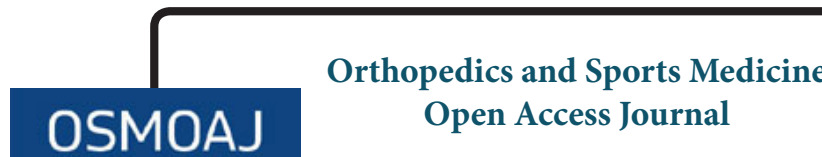

\section{Assets of Publishing with us}

- Global archiving of articles

- Immediate, unrestricted online access

- Rigorous Peer Review Process

- Authors Retain Copyrights

- Unique DOI for all articles 